Präv Gesundheitsf 2019 • 14:91

https://doi.org/10.1007/s11553-018-0685-7

Online publiziert: 9. November 2018

(c) Springer-Verlag GmbH Deutschland, ein Teil von Springer Nature 2018

CrossMark

Josef Weigl

Gesundheitsamt Plön, Schleswig-Holstein, Plön, Deutschland

\title{
Erratum zu: Die Sinnhaftigkeit von Schulbetretungsverboten für Ungeimpfte bei Masern- ausbrüchen und Alternativen
}

\section{Erratum zu:}

Präv Gesundheitsf 2018

https://doi.org/10.1007/s11553-018-

0676-8

Leider sind uns im oben genannten Artikel Fehler aufgefallen, die wir hiermit korrigieren möchten:

Zusammenfassung. Der zweite Satz des Abschnittes „Ergebnisse“ muss wie folgt lauten:

In beiden Fällen ist damit die Forderung einer Ansteckungswahrscheinlichkeit von über $50 \%$ nicht erfüllt und somit das Verhängen von Schulbetretungsverboten unrechtmäßig.

Ergebnisse. In Abschnitt „Kontrollbemühungen durch Impfen im Ausbruch“ fehlt im ersten Satz eine Klammer. Der Satz muss folgendermaßen lauten:

Andererseits steht mit den kombinierten Masern-Mumps-Röteln-(Varizellen-) Impfungen ein wirksames Instrument zur Verfügung. $M M R(V)$ sind Masernlebendimpfstoffe mit hervorragender Wirksamkeit (über 90\%) und lebenslanger Immunität, die zudem gut verträglich sind und als Routineimpfung ab dem 11. Lebensmonat zweimalig vorgesehen sind [5].

Fazit für die Praxis. Der erste Satz muss wie folgt lauten:

Die Ansteckungswahrscheinlichkeit bei nichtgeimpften Schülern ist durchweg unter $50 \%$.
Wir bitten diese Fehler zu entschuldigen. Der Originalbeitrag wurde korrigiert.

\section{Korrespondenzadresse}

\section{Josef Weigl}

Gesundheitsamt Plön, Schleswig-Holstein

Plön, Deutschland

josef.weigl@kreis-ploen.de 